\title{
ASYMPTOTIC PROPERTIES OF THE MAXIMUM IN A STATIONARY GAUSSIAN PROCESS
}

\author{
BY \\ JAMES PICKANDS III $\left({ }^{1}\right)$
}

1. Introduction. Let $\{X(t),-\infty<t<\infty\}$ be a separable measurable version of a stationary Gaussian stochastic process. It is assumed without loss of generality that for all $s, t,-\infty<s, t<\infty$

$$
E X(t)=0, \quad E X(s) X(s+t)=r(t),
$$

where $r(t)$ is the covariance function. By stationarity, of course, the latter does not depend upon $s$. It is assumed, throughout, that

$$
r(t)=1-C|t|^{\alpha}+o\left(|t|^{\alpha}\right)
$$

as $t \rightarrow 0$ for some $C, 0<C<\infty$, and some $\alpha, 0<\alpha \leqq 2$. This is sufficient to ensure that the realizations are continuous everywhere with probability 1 . See Belayev [1]. A covariance function cannot satisfy (1.2) with $\alpha>2$, since then, $r(t)$ would not be positive definite. In this paper, we investigate the random variables

$$
Z(t) \equiv \sup _{0 \leqq s \leqq t} X(s)
$$

and their behavior as $t \rightarrow \infty$.

In $\S 2$, the form of the asymptotic distribution of $Z(t)$ is given for all $\alpha$. This generalizes the result of [6] wherein $\alpha=1$, and the result of Cramér [4] and Volkonski and Rozanov [9], wherein $\alpha=2$. In $\S 3$, the almost sure asymptotic behaviour of $Z(t)$ is investigated for all $\alpha$. For the case $\alpha=2$, the result extends that of Shur [8].

The results of this work depend heavily upon some of the results involving upcrossings given in [7]. They are valid for smallest as well as for largest values, provided appropriate but obvious modifications are made.

2. The asymptotic distribution. The main result of this section is the following

THEOREM 2.1. If (1.1) and (1.2) hold, and either

$$
\lim _{t \rightarrow \infty} r(t) \log t=0,
$$

or

$$
\int_{-\infty}^{\infty} r^{2}(t) d t<\infty
$$

Received by the editors January 17, 1969 and, in revised form, February 24, 1969.

(1) Supported by the National Science Foundation Grant GP-8716.

Copyright (C) 1969, American Mathematical Society 
then for all $x,-\infty<x<\infty$,

$$
\lim _{t \rightarrow \infty} P\left\{(A(t))^{-1}(Z(t)-B(t)) \leqq x\right\}=\exp -e^{-x},
$$

where

$$
A(t) \equiv(2 \log t)^{-1 / 2}
$$

and

$$
B(t) \equiv(2 \log t)^{1 / 2}
$$

$$
+\left(\left(\frac{1}{2}-\frac{1}{\alpha}\right) \log \log t+\log \left((2 \pi)^{-1 / 2} C^{1 / \alpha} H_{\alpha} 2^{(2-\alpha) / 2 \alpha}\right)\right) /(2 \log t)^{1 / 2},
$$

and

$$
0<H_{\alpha} \equiv \lim _{T \rightarrow \infty} T^{-1} \int_{0}^{\infty} e^{s} P\left\{\sup _{0 \leqq t \leqq T} Y(t)>s\right\} d s<\infty
$$

where $Y(t)$ is a nonstationary Gaussian process with means and covariances;

$$
E Y(t)=-|t|^{\alpha}, \quad \operatorname{cov}\left(Y\left(t_{1}\right), Y\left(t_{2}\right)\right)=\left|t_{1}\right|^{\alpha}+\left|t_{2}\right|^{\alpha}-\left|t_{2}-t_{1}\right|^{\alpha} .
$$

Before proceeding to the proof, a lemma is presented which summarizes the results of Theorems 2.1 and 3.2 of [7].

LEMMA 2.1. Let $N(\varepsilon, y, t)$ be the number of " $\varepsilon$-upcrossings" of the level $y$ in the interval $(o, t)$. An " $\varepsilon$-upcrossing" of the level $y$ is said to have occurred at $t_{0}$ if $X\left(t_{0}\right)=x$, and $X(t)<x$, for all $t, t_{0}-\varepsilon \leqq t \leqq t_{0}$. Then under the conditions of Theorem 2.1 ,

$$
\lim _{y \rightarrow \infty} P\{N(\varepsilon, y, \lambda / \mu)=k\}=e^{-\lambda} \lambda^{k} / k !, \quad k=0,1,2, \ldots,
$$

where $\mu \equiv E N(\varepsilon, y, t) / t$, has the same value for all $t$. Furthermore

$$
\mu \sim(2 \pi)^{-1 / 2} C^{1 / \alpha} H_{\alpha} y^{(2-\alpha) / \alpha} \exp \left(-y^{2} / 2\right),
$$

as $y \rightarrow \infty$, where $H_{\alpha}$ is given by (2.6).

Proof of Theorem 2.1. Clearly

$$
\begin{aligned}
P\{N(\varepsilon, A(t) x+ & \left.B(t), t)=0, \sup _{-\varepsilon \leqq t \leqq 0} X(t) \leqq A(t) x+B(t)\right\} \\
& \leqq P\left\{(A(t))^{-1}(Z(t)-B(t)) \leqq x\right\}=P\{Z(t) \leqq A(t) x+B(t)\} \\
& \leqq P\{N(\varepsilon, A(t) x+B(t), t)=0\} .
\end{aligned}
$$

But

$$
\begin{aligned}
& P\left\{N(\varepsilon, A(t) x+B(t), t)=0, \sup _{-\varepsilon \leqq t \leqq 0} X(t) \leqq A(t) x+B(t)\right\} \\
& =P\{N(\varepsilon, A(t) x+B(t), t)=0\} \\
& \quad-P\left\{N(\varepsilon, A(t) x+B(t), t)=0, \sup _{-\varepsilon \leqq t \leqq 0} X(t)>A(t) x+B(t)\right\} \\
& \geqq P\{N(\varepsilon, A(t) x+B(t), t)=0\}-P\left\{\sup _{-\varepsilon \leqq t \leqq 0} X(t)>A(t) x+B(t)\right\} .
\end{aligned}
$$


However, $P\left\{\sup _{-\varepsilon \leqq t \leqq 0} X(t)>A(t) x+B(t)\right\} \rightarrow 0$, as $t \rightarrow \infty$, since $A(t) x+B(t) \rightarrow \infty$ as $t \rightarrow \infty$ for all real $x$, and, as observed in the introduction, the realizations are continuous everywhere with probability 1 . So it is sufficient to prove that

$$
\lim _{t \rightarrow \infty} P\{N(\varepsilon, A(t) x+B(t), t)=0\}=\exp -e^{-x}
$$

for all real $x$. But, by Lemma 2.1 , this is true, provided

$$
\lim _{t \rightarrow \infty} t \mu=e^{-x} .
$$

However $\mu \sim(2 \pi)^{-1 / 2} C^{1 / \alpha} H_{\alpha} y^{(2-\alpha) / \alpha} \exp \left(-y^{2} / 2\right)$, as $y \rightarrow \infty$, or equivalently as $t \rightarrow \infty$, where $y=A(t) x+B(t)$. Evidently $y^{(2-\alpha) / \alpha} \sim 2^{(2-\alpha) / 2 \alpha}(\log t)^{(2-\alpha) / 2 \alpha}$ as $t \rightarrow \infty$.

But

$$
\begin{aligned}
y^{2} / 2= & \left(x^{2} / 4 \log t\right)+\log t+\left(\left(D_{1} \log \log t+D_{2}\right)^{2} / 4 \log t\right)+x \\
& +\left(x\left(D_{1} \log \log t+D_{2}\right) / 2 \log t\right)+\left(D_{1} \log \log t+D_{2}\right) \\
= & \log t+x+D_{1} \log \log t+D_{2}+o(1) \quad \text { as } t \rightarrow \infty,
\end{aligned}
$$

where $D_{1} \equiv 1 / \alpha-1 / 2$, and $D_{2} \equiv \log \left((2 \pi)^{-1 / 2} C^{1 / \alpha} H_{\alpha} 2^{(2-\alpha) / \alpha}\right)$. So

$$
\exp \left(-y^{2} / 2\right) \sim t^{-1}(\log t)^{-D_{1} e^{-D_{2}} e^{-x}} \quad \text { as } t \rightarrow \infty,
$$

and

$$
t \mu \sim(2 \pi)^{-1 / 2} C^{1 / \alpha} H_{\alpha} 2^{(2-\alpha) / \alpha}(\log t)^{((2-\alpha) / 2 \alpha)-D_{1}} e^{-D_{2} e^{-x}}=e^{-x} \quad \text { as } t \rightarrow \infty .
$$

The theorem is proved.

When $\alpha=1$, it was shown in [7], using the results of [6], that $H_{1}=1$. Then, the result of Theorem 2.1 coincides with the result of Theorems 4.2 and 4.3 of [6], albeit under slightly weaker conditions.

When $\alpha=2$, it was shown in [7], using the results of [4] and [9], that $H_{2}=1 / \sqrt{ } \pi$. When this is the case the result of Theorem 2.1 coincides with those in [4] and [9], which are the same except that the conditions in [4] are weaker than those in [9]. The conditions considered in this work are of two kinds. Those involving the behaviour of $r(t)$ as $t \rightarrow 0$ are called "local conditions". An example is (1.2). Those concerning the behaviour of $r(t)$ as $t \rightarrow \infty$ are called "mixing conditions". Examples are (2.1) and (2.2). The conditions of both types are weaker in Theorem 2.1 than in [4].

It is natural to inquire whether the conditions of Theorem 2.1 might be further weakened. In particular, might the conditions (2.1) or (2.2) be replaced by the condition that

$$
\lim _{t \rightarrow \infty} r(t)=0
$$

For the case of stationary Gaussian sequences, a similar question was answered negatively in [6]. So it is strongly conjectured that (2.8) is not sufficient. 
The remarks at the end of $\S 4$ of [6] concern the conditions on the spectral distribution function which are sufficient for the conditions (2.1), (2.2), and (2.8). Conditions which are sufficient for (1.2) are given in $\$ 4$ of [7].

From the discussion at the end of $\$ 4$ of [6], it is clear that the strong mixing condition is sufficient for the result of Theorem 2.1.

3. The almost sure behaviour. In this section, asymptotic inequalities are given which hold with probability 1 . The main results are Theorems 3.1, 3.2 and 3.3. Theorem 3.3 simplifies the conditions of Theorem 3.2.

THEOREM 3.1. If $X(t)$ is such that (1.1) and (1.2) hold, and if

then

$$
\sup _{0 \leqq t \leqq 1}|t|^{-\alpha}\left(1-r^{2}(t)\right)>0,
$$

(3.1) $P\left\{\limsup _{t \rightarrow \infty}(2 \log t)^{1 / 2}\left(X(t)-(2 \log t)^{1 / 2}\right) / \log \log t \leqq \frac{1}{2}+\frac{1}{\alpha}\right\}=1$.

Note that this theorem requires no "mixing" condition. A "mixing" condition is one involving the behaviour of $r(t)$ as $t \rightarrow \infty$.

Before proceeding to the proof, a few lemmas are given.

LeMma 3.1. For all $x>0$,

$$
1-\Phi(x) \leqq \psi(x),
$$

where

$$
\Phi(x)=\frac{1}{\sqrt{ } 2 \pi} \int_{-\infty}^{x} \exp \left(-t^{2} / 2\right) d t
$$

and

$$
\psi(x) \equiv(2 \pi)^{-1 / 2} x^{-1} \exp \left(-x^{2} / 2\right)
$$

Furthermore

$$
\lim _{x \rightarrow \infty}(1-\Phi(x)) / \psi(x)=1 .
$$

The result (3.5) is shown in Cramér [3, p. 374]. The result (3.2) follows by differentiation.

LEMma 3.2. If $t$ is so chosen that

$$
\inf _{0 \leqq s \leqq t}|s|^{-\alpha}\left(1-r^{2}(s)\right)>0,
$$

and if (1.1) and (1.2) hold, then

$$
\lim _{x \rightarrow \infty} P\{Z(t)>x\} / x^{2 / \alpha} \psi(x) t=C^{1 / \alpha} H_{\alpha},
$$

where $\psi(x)$ is given by (3.4) and $H_{\alpha}$ by (2.6).

This is Lemma 2.9 of [7]. 
LEMMA 3.3. Let

$$
\psi(x, \gamma) \equiv x^{\gamma} \exp \left(-x^{2} / 2\right)
$$

and let

$$
x(\beta, t) \equiv(2 \log t)^{1 / 2}+\beta \log \log t /(2 \log t)^{1 / 2}
$$

Then

$$
\psi(x(\beta, t), \gamma) \sim 2^{\gamma / 2} t^{-1}(\log t)^{-\beta+\gamma / 2},
$$

as $t \rightarrow \infty$.

Proof. By definition (3.8), $x^{2}(\beta, t) / 2=\log t+\beta \log \log t+\beta^{2}(\log \log t)^{2} / 4 \log t$ $=\log t+\beta \log \log t+o(1)$, as $t \rightarrow \infty$. So

$$
\exp \left(-x^{2}(\beta, t) / 2\right) \sim t^{-1}(\log t)^{-\beta},
$$

as $t \rightarrow \infty$. Furthermore, $\log x(\beta, t)=\frac{1}{2} \log 2+\frac{1}{2} \log \log t+\log (1+\beta \log \log t / 2 \log t)$ $=\frac{1}{2} \log 2+\frac{1}{2} \log \log t+o(1)$, as $t \rightarrow \infty$. So

$$
x^{\gamma}(\beta, t) \sim 2^{\gamma / 2}(\log t)^{\gamma / 2},
$$

as $t \rightarrow \infty$. Combining (3.10) and (3.11), the result (3.9) follows. The lemma is proved.

Proof of Theorem 3.1. Clearly it is sufficient to prove that for any $\beta>(1 / 2+1 / \alpha)$, $X_{n} \geqq x(\beta, n)$, only a finite number of times with probability one, where $x(\beta, t)$ is given by (3.8), and $X_{n} \equiv \sup _{n-1 \leqq s \leqq n} X(s)$. By Lemmas 3.2 and 3.3, $P\left\{X_{n} \geqq x(\beta, n)\right\}$ $\sim 2^{\gamma / 2}(2 \pi)^{-1 / 2} C^{1 / \alpha} H_{\alpha} n^{-1}(\log n)^{-\beta+\gamma / 2}$, where $\gamma=2 / \alpha-1$. But then

$$
\sum_{n=1}^{\infty} P\left\{X_{n} \geqq x(\beta, n)\right\}<\infty \text { if }-\beta+1 / \alpha-1 / 2<-1,
$$

or equivalently, if $\beta>1 / \alpha+1 / 2$. The theorem is proved.

THEOREM 3.2. Assume that for every sufficiently small $\varepsilon>0$, there exists $a \gamma>1$ and $a \theta, 0<\theta<1$, such that

$$
\lim _{t \rightarrow \infty}(\log t)^{\gamma} D(\varepsilon, t)=0,
$$

where

$$
D(\varepsilon, t) \equiv \sum_{k=l}^{\left[x^{2 / \alpha} / \alpha\right]}\left(\left[x^{2 / \alpha} t / a\right]-k\right)\left|r\left(k a x^{-2 / \alpha}\right)\right| \exp -x^{2} /\left(1+\left|r\left(k a x^{-2 / \alpha}\right)\right|\right),
$$

where $[x]$ is the greatest integer less than or equal to $x$,

$$
l \equiv\left[x^{2 / \alpha} \theta / a\right]
$$

and

$$
x \equiv x\left(\left(-\frac{1}{2}+\frac{1}{\alpha}-\varepsilon\right), t\right)
$$


where $x(\beta, t)$ is given by (3.8). Assume also that (1.1) and (1.2) hold, and that

$$
\lim _{t \rightarrow \infty} r(t)=0
$$

Then

$$
P\left\{\liminf _{t \rightarrow \infty}(2 \log t)^{1 / 2}\left(Z(t)-(2 \log t)^{1 / 2}\right) / \log \log t \geqq-\frac{1}{2}+\frac{1}{\alpha}\right\}=1 .
$$

Before proceeding to the proof, a series of supporting lemmas is given.

LEMMA 3.4. Let $t(\varepsilon, m)$ be a real valued function of the positive real valued argument $\varepsilon$, and the integer valued argument $m$. Assume that for every sufficiently small $\varepsilon>0$,

$$
h(\varepsilon, t(\varepsilon, m)) \geqq h(2 \varepsilon, t(\varepsilon, m+1))
$$

for all sufficiently large $m$, where

$$
\begin{aligned}
h(\varepsilon, t) & \equiv x\left(\left(-\frac{1}{2}+\frac{1}{\alpha}-\varepsilon\right), t\right) \\
& =(2 \log t)^{1 / 2}+\left(-\frac{1}{2}+\frac{1}{\alpha}-\varepsilon\right) \log \log t /(2 \log t)^{1 / 2} .
\end{aligned}
$$

If, for every sufficiently small $\varepsilon>0, Z(t(\varepsilon, m)) \leqq h(\varepsilon, t(\varepsilon, m))$, only a finite number of times with probability 1, then (3.17) holds.

Proof. What is to be proved is that for any sufficiently small $\varepsilon>0$, with probability 1 there exists a $t_{0}$ which is such that

$$
Z(t)>h(\varepsilon, t)
$$

for any $t>t_{0}$. Let $\varepsilon>0$ be chosen arbitrarily but sufficiently small so that the condition (3.18) of the lemma is satisfied. By assumption, with probability 1 there exists an integer $m_{0}$ which is such that, for all $m \geqq m_{0}, Z(t(\varepsilon, m)) \geqq h(\varepsilon, t(\varepsilon, m))$. But then, $Z(t)$ is nondecreasing and for sufficiently large $t, x(t, \varepsilon)$ is nondecreasing in $t$. We can assume, without loss of generality, that $m_{0}$ is so large that $x(t, \varepsilon)$ is nondecreasing for all $t \geqq t\left(\varepsilon, m_{0}\right)$. Let $t$ be any real number greater than $t\left(\varepsilon, m_{0}\right)$, and let $m$ be such that $t(\varepsilon, m) \leqq t<t(\varepsilon, m+1)$. Clearly $m \geqq m_{0}$. Then $Z(t) \geqq Z(t(\varepsilon, m))$ $\geqq h(\varepsilon, t(\varepsilon, m)) \geqq h(2 \varepsilon, t(\varepsilon, m+1)) \geqq h(2 \varepsilon, t)$. So, the result (3.20) holds, provided $\varepsilon$ is replaced by $2 \varepsilon$. But $\varepsilon$ was arbitrarily chosen. The lemma is proved.

LEMMA 3.5. The condition (3.18) of Lemma 3.4 is satisfied if

$$
t(\varepsilon, m)=\exp \varepsilon m \text {. }
$$

Proof. It is to be proved that

$$
h(\varepsilon, t(\varepsilon, m))-h(2 \varepsilon, t(\varepsilon, m+1)) \geqq 0,
$$

where $h(\varepsilon, t)$ is given by (3.19). But

$$
h(\varepsilon, t(\varepsilon, m))-h(2 \varepsilon, t(\varepsilon, m+1))=\sum_{i=1}^{3} D_{i}
$$


where

and

$$
\begin{aligned}
& D_{1} \equiv(2 \log t(\varepsilon, m))^{1 / 2}-(2 \log t(\varepsilon, m+1))^{1 / 2} \\
& D_{2} \equiv\left(\frac{1}{2}-\frac{1}{\alpha}+\varepsilon\right)\left((2 \log t(\varepsilon, m+1))^{-1 / 2} \log \log t(\varepsilon, m+1)\right. \\
& \left.\quad-(2 \log t(\varepsilon, m))^{-1 / 2} \log \log t(\varepsilon, m)\right),
\end{aligned}
$$

$$
D_{3} \equiv \varepsilon(2 \log t(\varepsilon, m+1))^{-1 / 2} \log \log t(\varepsilon, m+1) .
$$

First, consider the term $D_{1}$. Clearly $(m+1)^{1 / 2}-m^{1 / 2} \sim \frac{1}{2} m^{-1 / 2}$ as $t \rightarrow \infty$. So

$$
D_{1} \sim-\frac{1}{2}(2 \varepsilon / m)^{1 / 2}
$$

as $m \rightarrow \infty$. Consider the term $D_{2}$. Observe that $D_{2} \sim\left(\frac{1}{2}-(1 / \alpha)+\varepsilon\right)(g(m+1)-g(m))$, as $m \rightarrow \infty$, where $g(m)=(2 \varepsilon m)^{-1 / 2} \log \varepsilon m$. So for sufficiently large $m$,

$$
D_{2} \sim(2 \varepsilon)^{-1 / 2}\left(\frac{1}{2}-\frac{1}{\alpha}+\varepsilon\right)\left(-\frac{1}{2} m^{-3 / 2} \log \varepsilon m+m^{-3 / 2}\right)
$$

as $m \rightarrow \infty$. On the other hand

$$
D_{3}=\varepsilon(2 \varepsilon(m+1))^{-1 / 2} \log \varepsilon(m+1)
$$

which is, asymptotically, the dominant term. So by (3.23) through (3.26), the result follows. The lemma is proved.

LEMMA 3.6. In order that (3.17) hold, it is sufficient that for every sufficiently small $\varepsilon>0$, there exists a $\gamma>1$ such that

$$
\lim _{t \rightarrow \infty}(\log t)^{\gamma} P\{Z(t) \leqq h(\varepsilon, t)\}=0 .
$$

Proof. By Lemmas 3.4 and 3.5 and the Borel Cantelli Lemma (Loeve [5, p. 228]), it is sufficient to show that for every sufficiently small $\varepsilon>0$,

$$
\sum_{m=1}^{\infty} P\{Z(t(\varepsilon, m)) \leqq h(\varepsilon, t(\varepsilon, m))\}<\infty ;
$$

where $h(\varepsilon, t)$ is given by (3.19) and $t(\varepsilon, m)$ by (3.21). It is sufficient that for any $\varepsilon>0$, for some $\gamma>1, \lim _{m \rightarrow \infty} m^{\gamma} P\{Z(t(\varepsilon, m)) \leqq h(\varepsilon, t(\varepsilon, m))\}=0$. Equivalently, by (3.21) the condition (3.27) is sufficient. The lemma is proved.

Lemma 3.7. Let $P(\cdot)$ and $P^{\prime}(\cdot)$ be two multivariate Gaussian measures assigning means 0 , variances 1 , and covariances $r_{i j}$ and $r_{i j}^{\prime}$ respectively. Then, for any real finite $c$,

$$
\begin{aligned}
\mid P\left\{\max _{1 \leqq i \leqq n} X_{i} \leqq c\right. & -P^{\prime}\left\{\max _{1 \leqq i \leqq n} X_{i} \leqq c\right\} \mid \\
& \leqq D_{n}=\sum_{i, j=1}^{n}\left(1-\left(r_{i j}^{\prime \prime}\right)^{2}\right)^{-1 / 2}\left|r_{i j}-r_{i j}^{\prime}\right| \exp -c^{2} /\left(1+\left|r_{i j}^{\prime \prime}\right|\right)
\end{aligned}
$$

where $r_{i j}^{\prime \prime} \equiv \max \left(r_{i j}, r_{i j}^{\prime}\right)$. 
This lemma was proved by Berman [2], who expressed it in terms of stationary covariance sequences. The present formulation was given by the author in [6].

LEMMA 3.8. For any $a, t, 0<a, t<\infty$, there exists a real finite positive constant $H_{\alpha}(a)$ such that

$$
\lim _{x \rightarrow \infty} P\left\{Z_{x}(t)>x\right\} / x^{2 / \alpha} \psi(x) t=C^{1 / \alpha} H_{\alpha}(a) / a,
$$

where $\psi(x)$ is given by (3.4) and

$$
\begin{aligned}
Z_{x}(t) & \equiv Z_{x}(o, t), \\
Z_{x}\left(b_{1}, b_{2}\right) & \equiv \max _{\left[b_{1} x^{2 / \alpha} / a\right] \leqq i \leqq\left[b_{2} x^{2 / \alpha} / a\right]} X\left(\operatorname{iax}^{-2 / \alpha}\right),
\end{aligned}
$$

provided (1.1) and (1.2) hold and

$$
\inf _{0 \leqq s \leqq t}|s|^{-\alpha}(1-r(s))>0 .
$$

This is Lemma 2.5 of [7].

Proof of Theorem 3.2. Let $\varepsilon>0$ be arbitrarily chosen and let $\theta, 0<\theta<1$ be chosen arbitrarily but so that the condition (3.12) is satisfied. Let

$$
Z_{x}^{\prime}(t) \equiv \max _{1 \leqq k \leqq[t]} Z_{x}(k-1+\theta, k) \text {. }
$$

Clearly, $Z(t)$ is stochastically larger than $Z_{x}^{\prime}(t)$. That is for any $x, P\{Z(t) \leqq x\}$ $\leqq P\left\{Z_{x}^{\prime}(t) \leqq x\right\}$. So, by Lemma 3.6, it is sufficient to prove that for some $\gamma>1$,

$$
\lim _{t \rightarrow \infty}(\log t)^{\gamma} P\left\{Z_{x}^{\prime}(t) \leqq h(\varepsilon, t)\right\}=0,
$$

where $x=h(\varepsilon, t)$, which is given by (3.19). The proof proceeds in two stages. First, it is shown that for some $\gamma>1$,

$$
\lim _{t \rightarrow \infty}(\log t)^{\gamma} P^{\prime}\left\{Z_{x}^{\prime}(t) \leqq h(\varepsilon, t)\right\}=0,
$$

where $P^{\prime}(\cdot)$ is the measure which confers independence among the successive half open (on the left) unit intervals, but is otherwise identical to $P^{\prime}(\cdot)$. More specifically, let $\mathscr{F}_{k}$ be the subsigma field generated by $(X(t), k-1<t \leqq k)$. On every subsigma field $\mathscr{F}_{k}, P^{\prime}(\cdot)=P(\cdot)$, but on $\mathscr{F}$, which is generated by $(X(t),-\infty<t<\infty), P^{\prime}(\cdot)$ is the product measure. The second part consists in proving that for some $\gamma>1$,

$$
\lim _{t \rightarrow \infty}(\log t)^{\gamma}\left(P\left\{Z_{x}^{\prime}(t) \leqq h(\varepsilon, t)\right\}-P^{\prime}\left\{Z_{x}^{\prime}(t) \leqq h(\varepsilon, t)\right\}\right)=0,
$$

where $x=h(\varepsilon, t)$, which is given by (3.19).

First, let us prove (3.32). By the definition of $P^{\prime}(\cdot)$,

$$
P^{\prime}\left\{Z_{x}(t) \leqq h(\varepsilon, t)\right\} \leqq \prod_{i=1}^{[t]} P\left\{Z_{x}(k-1+\varepsilon, k) \leqq h(\varepsilon, t)\right\} .
$$

So $-\log P^{\prime}\left\{Z_{x}^{\prime}(t) \leqq h(\varepsilon, t)\right\} \geqq-[t] \log P\left\{Z_{x}(k-1+\varepsilon, k) \leqq h(\varepsilon, t)\right\}$. But, if $F(x)$ is any cumulative distribution function, $-\log F(x)=-\log (1-(1-F(x)))=(1-F(x))$ $+O(1-F(x))^{2}$, as $F(x) \rightarrow 1$. The condition (3.16) together with (1.2) implies that 
the condition (3.31) of Lemma 3.8 is satisfied. Taken together with Lemma 3.3, this implies that there exists a positive finite constant $C_{1}$ which is such that $-\log P^{\prime}\left\{Z_{x}(t) \leqq h(\varepsilon, t)\right\} \geqq C_{1} t^{-1}(\log t)^{\varepsilon}$, where $x=h(\varepsilon, t)$. Thus

$$
-\log P^{\prime}\left\{Z_{x}^{\prime}(t) \leqq h(\varepsilon, t)\right\} \geqq C_{1}(\log t)^{\varepsilon} .
$$

That is $P^{\prime}\left\{Z_{x}(t) \leqq h(\varepsilon, t)\right\} \leqq \exp -C_{1}(\log t)^{\varepsilon}$ as $t \rightarrow \infty$. So (3.32) holds.

Now (3.33) is proven. By the condition (3.16), and the fact that intervals of width $\varepsilon$ are "chopped out", the term $\left(1-r_{i j}^{2}\right)^{-1 / 2}$ can be replaced by a constant in applying Lemma 3.7. The latter, however, establishes that the condition (3.12) implies (3.33). The theorem is proved.

THEOREM 3.3. If either

$$
\lim _{t \rightarrow \infty} t^{\lambda} r(t)=0
$$

for some $\lambda>0$, or

$$
\int_{-\infty}^{\infty} r^{2}(t) d t<\infty
$$

and if (1.1) and (1.2) hold, then (3.17) holds.

Before proving the theorem, a few lemmas are given.

LEMMA 3.9. Simultaneously for all $m \geqq 1$, if (1.1) and (1.2) hold, for any $a, 0<a<\infty$,

$$
\frac{1}{m} \sum_{k=1}^{m}\left|r\left(k a x^{-2 / \alpha}\right)\right|=\frac{1}{T} \int_{0}^{T}|r(t)| d t+o\left(a x^{-2 / \alpha}\right)
$$

as $x \rightarrow \infty$, where $T=\max ^{-2 / \alpha}$.

This is Lemma 3.6 of [7].

Lemma 3.10. In order that the condition (3.12) hold, it is sufficient that

$$
\lim _{T \rightarrow \infty} T^{\lambda-1} \int_{0}^{T}|r(t)| d t=0
$$

for some $\lambda>0$, and

$$
\lim _{t \rightarrow \infty} r(t)=0 .
$$

Proof. Observe that $\exp \left(-x^{2} /(1+r)\right)=\left(\exp \left(-x^{2} / 2\right)\right)^{2 /(1+r)}$, that, by (3.19),

$$
x \sim(2 \log t)^{1 / 2}
$$

as $t \rightarrow \infty$, and by Lemma 3.3, exp $-x^{2} / 2 \sim t^{-1}(\log t)^{-\beta}$, where $\beta \equiv\left(-\frac{1}{2}+1 / \alpha-\varepsilon\right)$. But

$$
\begin{aligned}
\left(\left[x^{2 / \alpha} t / a\right]-k\right) \exp \left(-x^{2} /(1+r)\right) & \leqq\left[x^{2 / \alpha} t / a\right] \exp \left(-x^{2} /(1+r)\right) \\
& \sim 2^{1 / \alpha} a^{-1} t(\log t)^{1 / \alpha} t^{-2 /(1+r)}(\log t)^{-2 \beta /(1+r)} \\
& \leqq 2^{1 / \alpha} a^{-1} t(\log t)^{1 / \alpha} t^{-2 /(1+r)} \\
& =2^{1 / \alpha} a^{-1} t^{-1}(\log t)^{1 / \alpha} t^{2 r /(1+r) \quad \text { as } t \rightarrow \infty}
\end{aligned}
$$


So

$(\log t)^{\gamma} D(\varepsilon, t) \sim 2^{1 / \alpha} a^{-1} t^{-1}(\log t)^{\gamma+1 / \alpha} \sum_{k=l}^{[x / \alpha t / \alpha]}\left|r\left(k a x^{-2 / \alpha}\right)\right| t^{2|r(k a x-2 / \alpha)| /(1+|r(k a x-2 / \alpha)|)}$

where $l$ is given by (3.14). Let $t_{0}$ be an arbitrary positive finite real number. Then

$$
(\log t)^{\gamma} D(\varepsilon, t) \equiv D_{1}(\varepsilon, t)+D_{2}(\varepsilon, t),
$$

where $D_{1}(\varepsilon, t)$ is the same as $(\log t)^{\gamma} D(\varepsilon, t)$ except that the sum is from $l$ to $\left[x^{2 / \alpha} t_{0} / a\right]$. By (3.37), all of the terms $\left|r\left(\operatorname{kax}^{-2 / \alpha}\right)\right|$ are less than $\delta(\theta)$, where, for any $s, 0<s<\infty$, by (3.37), $\delta(s) \equiv \sup _{s \leqq t<\infty} 2|r(s)| /(1+|r(s)|)<1$. So for any fixed $t_{0}$, and any $C_{1}, 2^{1 / \alpha} a^{-1}<C_{1}<\infty$,

$$
D_{1}(\varepsilon, t) \leqq C_{1} \delta_{1}(\theta)(\log t)^{\gamma+1 / \alpha} t^{(2 \delta(\theta) /(1+\delta(\theta)))-1} x^{2 / \alpha} / a
$$

for all sufficiently large $t$. So

$$
\lim _{t \rightarrow \infty} D_{1}(\varepsilon, t)=0 .
$$

Let $\lambda>0$ be chosen arbitrarily, but so that the condition (3.36) is satisfied, and let $t_{0}$ be so chosen that $\delta\left(t_{0}\right)<\lambda$. By (3.39), it is sufficient to prove that $\lim _{t \rightarrow \infty} D_{2}(\varepsilon, t)$ $=0$. But, clearly, there exists a constant $C_{2}$ such that

$$
\begin{aligned}
D_{2}(\varepsilon, t) & \leqq C_{1}(\log t)^{\gamma+1 / \alpha} t^{\lambda-1} \sum_{k=l}^{[x / \alpha / a]}\left|r\left(k a x^{-2 / \alpha}\right)\right| \\
& \leqq C_{2}(\log t)^{\gamma+2 / \alpha} t^{\lambda}\left(x^{2 / \alpha} t / a\right)^{-1} \sum_{k=l}^{\left[x^{2 / \alpha} / a\right]}\left|r\left(k a x^{-2 / \alpha}\right)\right| .
\end{aligned}
$$

But, by Lemma 3.9,

$$
\limsup _{t \rightarrow \infty}\left(x^{2 / \alpha} T / a\right)^{-1} \sum_{k=l}^{\left[x^{2 / \alpha} T / a\right]}\left|r\left(k a x^{-2 / \alpha}\right)\right| /\left(T^{-1} \int_{0}^{T}|r(t)| d t\right) \leqq 1 .
$$

So, it is sufficient that there exist a $\lambda^{\prime}>0$ such that

$$
\lim _{T \rightarrow \infty} T^{\lambda^{\prime}-1}(\log T)^{\gamma+2 / \alpha} \int_{0}^{T}|r(t)| d t=0
$$

and that (3.37) hold. But for any $\lambda^{\prime}>0$ which satisfies (3.40), there is a $\lambda>0$ which satisfies (3.36). The lemma is proved.

Lemma 3.11. If (3.35) holds, then (3.37) does.

This is Lemma 4.8 of [6].

Proof of Theorem 3.3. By Theorem 3.2 and Lemma 3.10, it is sufficient to prove that the conditions (3.36) and (3.37) hold, if either (3.34) or (3.35) does. By Lemma 3.11, if (3.35) holds then (3.37) does. Clearly (3.34) implies (3.37). Now it must be proved that for some $\lambda>0,(3.36)$ holds.

First assume that (3.34) holds. In particular, let $\lambda$ be so chosen that it does. Let 
$\varepsilon>0$ be arbitrarily chosen. Then there exists a $t_{0}$, such that if $t \geqq t_{0}, r(t) \leqq \varepsilon t^{-\lambda}$ Clearly

$$
T^{\lambda-1} \int_{0}^{T}|r(t)| d t=T^{\lambda-1} \int_{0}^{t_{0}}|r(t)| d t+T^{\lambda-1} \int_{t_{0}}^{T}|r(t)| d t .
$$

But, if $\lambda<1$, as can be assumed without loss of generality,

$$
\lim _{T \rightarrow \infty} T^{\lambda-1} \int_{0}^{t_{0}}|r(t)| d t=0
$$

Consider the second term on the right side of (3.41).

$$
\begin{aligned}
T^{\lambda-1} \int_{t_{0}}^{T}|r(t)| d t & \leqq \varepsilon T^{\lambda-1} \int_{t_{0}}^{T} t^{-\lambda} d t \\
& =\varepsilon T^{\lambda-1}\left(T^{1-\lambda}-t_{0}^{1-\lambda}\right)(1-\lambda)^{-1} \rightarrow \varepsilon(1-\lambda)^{-1}, \text { as } T \rightarrow \infty,
\end{aligned}
$$

provided $\lambda<1$. But $\varepsilon>0$ was arbitrarily chosen. So (3.34) is sufficient for (3.35).

Now assume that (3.35) holds. By the Cauchy-Schwarz inequality

$$
T^{\lambda-1} \int_{0}^{T}|r(t)| d t \leqq T^{\lambda}\left(T^{-1} \int_{0}^{T} r^{2}(t) d t\right)^{1 / 2} \rightarrow 0, \quad \text { as } t \rightarrow \infty
$$

if $\lambda<1 / 2$. The theorem is proved.

Theorems 3.1 and 3.3 can be combined to give the following.

COROllary 3.1. If (1.1) and (1.2) hold, and either (3.34) or (3.35) holds, then $P\left\{\liminf _{t \rightarrow \infty}(2 \log t)^{1 / 2}\left(Z(t)-(2 \log t)^{1 / 2}\right) / \log \log t\right.$

$$
\left.\geqq \frac{1}{\alpha}-\frac{1}{2}, \limsup _{t \rightarrow \infty}(2 \log t)^{1 / 2}\left(Z(t)-(2 \log t)^{1 / 2}\right) / \log \log t \leqq \frac{1}{\alpha}+\frac{1}{2}\right\}=1,
$$

where $Z(t)$ is given by (1.3).

Proof. Observe that $x(\beta, t)$ given by (3.8) is monotonically increasing. So $X(t)$ is bounded by $x(\beta, t)$ for all sufficiently large $t$ if and only if $Z(t)$ is. So $X(t)$ can be replaced by $Z(t)$ in Theorem 3.1. Obviously (3.34) implies (3.37). By Lemma 3.11 (3.35) implies it as well. This is sufficient to guarantee that

$$
\inf _{0 \leqq t \leqq 1}|t|^{-\alpha}\left(1-r^{2}(t)\right)>0 .
$$

The corollary is proved.

The remarks at the end of $\$ 2$ pertain to this section as well. In fact, all of the conditions which have been found to be sufficient for (2.1) are sufficient for (3.34) as well.

For the case $\alpha=2$, the result of Theorem 3.1 is the same as that given by Shur [8]. The result of Theorem 3.3 is stronger. His conditions are the same as those given by Cramér [4], which are the same as the condition (3.34). 
It is strongly conjectured that the inequality signs can be replaced by equality signs in the results of Corollary 3.1. Watanabe asserts that this is so in the first of these inequalities as a corollary to a very interesting result which he announces in [10]. The referee has pointed out that the mutual singularity of any two processes having different values of either $\alpha$ or $C$, is an immediate corollary of this conjecture.

\section{REFERENCES}

1. Yu. K. Belayev, Continuity and Hölder's conditions for sample functions of stationary Gaussian processes, Proc. Fourth Berkeley Symposium on Mathematical Statistics and Probability, Vol. II (1961), pp. 23-33.

2. S. M. Berman, Limit theorems for the maximum term in stationary sequences, Ann. Math. Statist. 35 (1964), 502-516.

3. H. Cramér, Mathematical methods of statistics, Princeton Univ. Press, Princeton, N. J., 1951.

4. - On the intersections between the trajectories of a normal stationary stochastic process and a high level, Ark. Mat. 20 (1965), 337-349.

5. M. Loeve, Probability theory, Van Nostrand, Princeton, N. J., 1963.

6. J. Pickands, Maxima of stationary Gaussian processes, Z. Wahrscheinlichkeitstheorie und Verw. Gebiete 7 (1967), 190-223.

7. —, Upcrossing probabilities for stationary Gaussian processes, Trans. Amer. Math. Soc. 145 (1969), 51-73.

8. M. G. Shur, On the maximum of a Gaussian stationary process, Theor. Probability Appl. 10 (1965), 354-357.

9. V. A. Volkonski, and Yu. A. Rozanov, Some limit theorems for random functions. II, Theor. Probability Appl. 6 (1961), 186-198.

10. H. Watanabe, An asymptotic property of Gaussian stationary processes, Proc. Japan Acad. 44 (1968), 895-896.

Virginia Polytechnic Institute, BLACKSBURG, Virginia 